\title{
Cromatografia líquido-sólido aplicada à análise de petróleo
}

\author{
Carol H. Collins \\ Instituto de Química, Universidade Estadual de Campinas, \\ Cep 13083-970, Campinas, SP, Brasil \\ e-mail:chc@iqm.unicamp.br
}

\section{Resumo}

Este artigo, sobre os "Pilares da Cromatografia”, descreve as atividades na área de análise de petróleo, iniciadas no fim do século XIX, principalmente as que aplicavam processos similares ao da cromatografia líquidosólido (CLS) antes que os trabalhos de Tswett se tornassem conhecidos. Esses estudos continuaram até a metade do século XX, quando outras técnicas de separação começaram a substituir a CLS nas determinações.

Palavras chave

História de cromatografia; cromatografia líquido-sólido; análise de petróleo.

\section{Liquid-solid chromatography applied to the analysis of petroleum}

\section{Abstract}

This chapter of "Pillars of Chromatography" describes the activities in the area of petroleum analysis that started at the end of the $19^{\text {th }}$ century, with emphasis on those that applied processes similar to those of liquidsolid chromatography (LSC) even before the work of Tswett was well known. These efforts continued until the middle of the $20^{\text {th }}$ century when other separation techniques began to substitute determinations made by LSC.

Keywords

History of chromatography; liquid-solid chromatography; Petroleum analysis.

Com a descoberta do petróleo em várias partes do mundo, iniciaram-se as aplicações desse novo material. Separações em frações com diversas aplicações foram feitas por diferentes processos. Um dos produtos importantes foi a vaselina, que em 1870, era produzida a partir de óleo mineral, requerendo uma etapa de descoramento, comumente realizada misturando-a crua com pó de carvão ou uma argila. Essa mistura era, então, submetida à destilação com vapor de água para fornecer o produto branqueado. Em 1886, Carl Engler (1842-1925) (Figura 1), professor titular de Tecnologia Química na Universidade Técnica (Technische Hochschule) de Karlsruhe, Alemanha, e seu aluno M. Böhm descreveram um processo alternativo, no qual a vaselina crua era forçada a passar através uma série de seis tubos, cada um de $75 \mathrm{~cm}$ de altura, com diâmetro interno de $5 \mathrm{~cm}$, contendo em torno de $1 \mathrm{~kg}$ de carvão ativado e aquecido por um invólucro por onde passava vapor de água ${ }^{1}$. Os autores indicaram que o produto que saía do último tubo apresentava uma massa específica menor que a do material original e era enriquecido em hidro- 


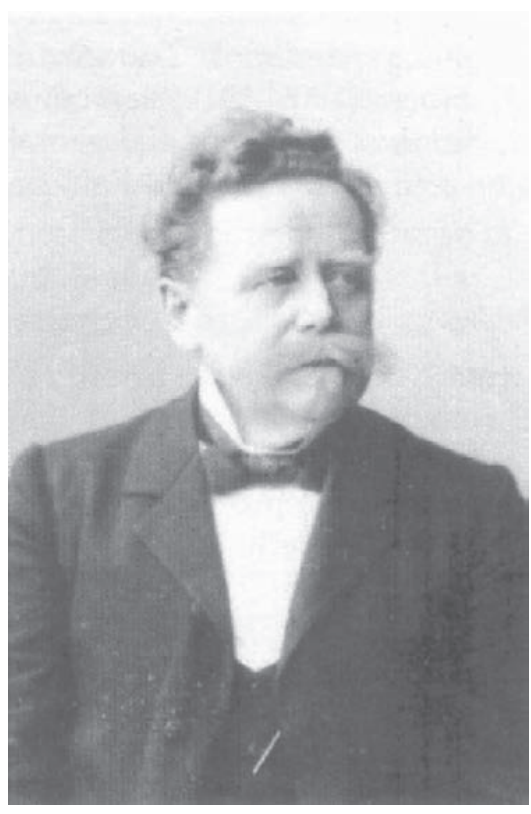

Figura 1 Foto de Carl Engler.

carbonetos saturados ${ }^{2}$. Parece um procedimento similar ao da cromatografia, entretanto os autores não estavam interessados em separações para fins de identificação e, sim, em um processo que pudesse remover as impurezas coloridas.

Um pesquisador da Inspeção Geológica dos Estados Unidos (U. S. Geological Survey), órgão responsável pelas pesquisas sobre recursos minerais, incluindo a área de petróleo, David Talbot Day (1859-1915), (Figura 2), iniciou, no fim da década de 1890, experimentos para verificar sua teoria sobre a origem dos petróleos que estavam sendo retirados de poços nos estados de Ohio e Pensilvânia, uma vez que apresentavam propriedades físicas diferentes. Ele propôs, em uma palestra apresentada à Sociedade Americana de Filosofia, em $1897^{3}$, que as pequenas diferenças nas propriedades podiam ser atribuídas à demora de alguns componentes em atravessarem os depósitos do petróleo. Ele considerou que os depósitos de Ohio, cujo petróleo tinha densidade maior, eram mais antigos do que os depósitos da Pensilvânia, os quais tinham perdido alguns componentes por "filtração" durante sua passagem

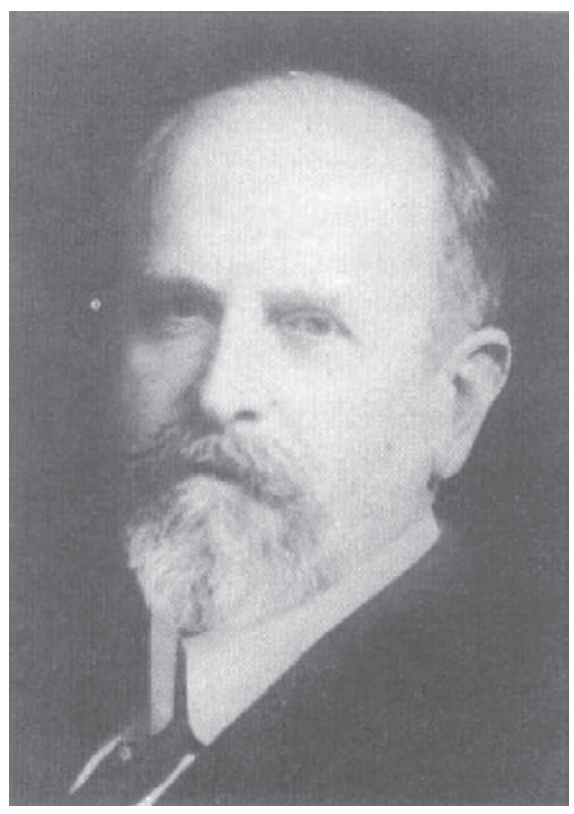

Figura 2 Foto de D.T. Day.

horizontal através das argilas do solo profundo. Ele também sugeriu que o petróleo obtido nos poços mais rasos, também menos denso, tinha perdido os seus componentes mais pesados durante a sua passagem na direção vertical nas argilas. Declarou que estava realizando experimentos que provavelmente comprovariam essa teoria, entretanto não apresentou nenhum resultado.

A mesma teoria foi apresentada por Day no primeiro Congresso Mundial sobre Petróleo (Congrès International du Pétrole), realizado em agosto de 1900, em Paris, França. Day descreveu as diferentes propriedades físicas de diversos petróleos, obtidos em Ohio e na Pensilvânia e indicou que diferentes argilas, existentes nesses estados e em outros, especialmente a terra de pisoeiro (fuller's earth, também chamada Florida earth, devido ao estado de origem), afetavam a passagem de tais amostras de maneiras diferentes $^{4}$. Entretanto, novamente, nenhum dado foi apresentado. Por outro lado, durante sua palestra em Paris, Day indicou que separações sem a necessidade de destilação seriam possíveis aplicando o método de "filtração". 
Um pesquisador presente nas palestras de Day foi o Prof. Engler. Ouvindo essa teoria, ele planejou alguns experimentos para verificar se os mesmos resultados seriam obtidos com as amostras de petróleo que possuia. Construiu, com a ajuda de seu colaborador Albrecht, um sistema para verificar a diferença entre os componentes de petróleo durante a sua passagem por argilas. Tal sistema (Figura 3) utilizava um tubo de vidro com $4 \mathrm{~cm}$ de diâmetro interno (C), recheado de 80 a $90 \mathrm{~cm}$ de altura com terra de pisoeiro, conectado a um reservatório, mantido a uma certa altura (A) por um tubo de vidro (B) e uma peça de borracha ao tubo com a argila. A amostra foi colocada no reservatório, e a força da gravidade permitiu a sua passagem através do tubo na maneira ascendente, sendo frações coletadas em um frasco (E). Os resultados do experimento são mostrados na Tabela 1 e confirmavam que as diferentes frações apresentavam propriedades diferentes ${ }^{5}$.

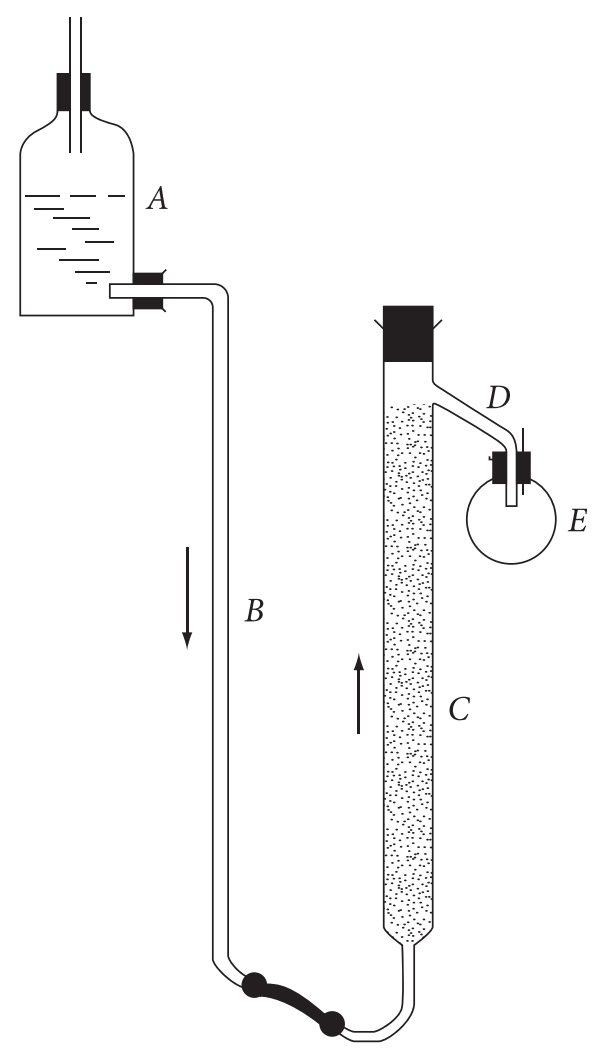

Figura 3 Sistema mais simples usado por Engler e Albrecht para a "filtração" de petróleo 5 .
Para entender melhor essa diferenciação, foi construído outro sistema (Figura 4), que permitiu verificar a composição do líquido durante a sua passagem pelo tubo, recheado com a terra de pisoeiro. O tubo continha seis saídas, separadas por $15 \mathrm{~cm}$. Nesse experimento, a amostra foi aplicada continuamente no tubo, até que o líquido saísse da posição 1. A partir desse momento, as outras saídas foram abertas, e o líquido foi coletado de cada ponto. Os resultados estão na Tabela 2 e novamente confirmaram que a "filtração" através da terra de pisoeiro fracionava alguns componentes da amostra original ${ }^{5}$.

Engler e Albrecht verificaram que o retardamento de alguns componentes não havia sido causado por uma reação química dos componentes ácidos do petróleo com os sítios básicos na argila e propuseram que a subida mais rápida dos componentes mais leves do petróleo devia-se à ação capilar, proposta por Goppelsröder ${ }^{6}$ para justificar as separações por ele conseguidas com tiras de papel colocadas em diversas misturas ${ }^{7}$.

Nos Estados Unidos, Joseph E. Gilpin (1866-1924) (Figura 5), professor de Química da Universidade de John Hopkins, em Baltimore, estado de Maryland, onde Day estudou, interessou-se pela teoria de Day. Após algumas discussões com Day $^{8}$, Gilpin e seus alunos realizaram diversos experimentos e, a partir de 1908, publicaram três trabalhos contendo várias tabelas de dados ${ }^{9-11}$. Nesses trabalhos, foram colocadas figuras que mostravam o equipamento utilizado (Figura 6). As amostras de petróleo foram colocadas em latas metálicas com volume em torno de um litro (A), e a passagem pelos tubos metálicos (B) foi induzida por vácuo (D), conectado através de uma lata grande $(\mathrm{C})$ para equalizar a pressão. Os tubos metálicos, com diâmetros internos de $2,8 \mathrm{~cm}$ e comprimentos de $170 \mathrm{~cm}$, foram recheados com terra de pisoeiro, inicialmente seca. A subida do petróleo pelo tubo foi lenta, 
Tabela 1 Algumas propriedades físico-químicas de frações de uma amostra de petróleo cru, coletadas nos frascos na saída do sistema mostrado na Figura $3^{[5]}$.

\begin{tabular}{|c|c|c|c|}
\hline $\begin{array}{l}\text { Número da } \\
\text { fração }\end{array}$ & $\begin{array}{l}\text { Volume da } \\
\text { fração }(m L)^{a}\end{array}$ & $\begin{array}{l}\text { Massa específica } \\
\text { a } 15^{\circ} \mathrm{C}\left(\mathrm{g} \cdot \mathrm{g}^{-1}\right)^{\mathrm{b}}\end{array}$ & Descrição da fração \\
\hline 1 & 70 & 0,7812 & Clara sem cor \\
\hline 2 & 65 & 0,7870 & Clara com leve fluorescência \\
\hline 3 & 120 & 0,7897 & Clara com leve fluorescência \\
\hline 4 & 70 & 0,7905 & Clara com leve fluorescência \\
\hline 5 & 50 & 0,7913 & Clara com leve fluorescência \\
\hline 6 & 110 & 0,7919 & Levemente amarelo com fluorescência verde \\
\hline 7 & 100 & 0,7920 & Mais amarelo \\
\hline 8 & 400 & 0,7965 & Amarelo com fluorescência verde \\
\hline 9 & 50 & 0,8020 & Amarelo com fluorescência verde \\
\hline 10 & 100 & 0,8032 & Amarelo com fluorescência verde \\
\hline 11 & 300 & 0,7976 & Laranja com fluorescência verde \\
\hline 12 & 500 & 0,7962 & Laranja com fluorescência verde \\
\hline
\end{tabular}

a Os volumes foram arbitrários; ${ }^{b}$ Massa específica do óleo inicial: 0,7929 (15 $\left.{ }^{\circ} \mathrm{C}\right)$.

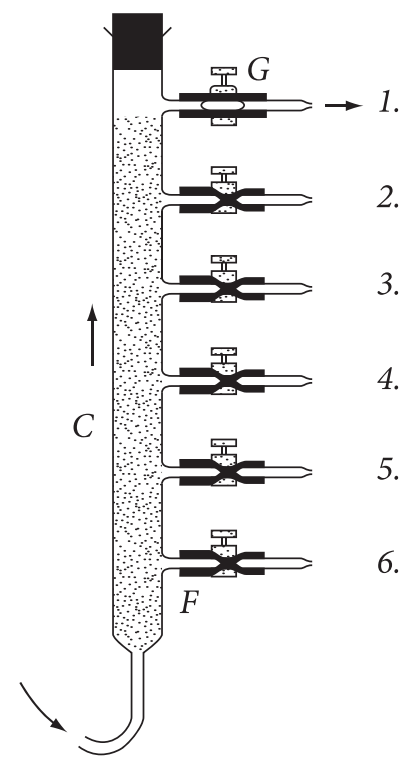

Figura 4 Outro sistema usado por Engler e Albrecht para a "filtração" de petróleo 5 .

gastando de sete a dez dias para atingir $75 \mathrm{~cm}$ na temperatura ambiente e três dias em uma temperatura de $70{ }^{\circ} \mathrm{C}$. Terminando o processo, os tubos foram desconectados e invertidos, e a argila foi cuidadosamente removida do tubo, cortada em secções que foram colocadas em tubos de vidro para extrair o líquido com água, permitindo que suas propriedades físico-químicas fossem determinadas (um exemplo está na Tabela 3). Para algumas frações, os seus conteúdos de enxofre e nitrogênio também foram determinados.

Os resultados indicaram que as frações próximas à saída do tubo continham hidrocarbonetos mais leves e que compostos aromáticos e insaturados subiram menos na argila. Os compostos contendo enxofre e nitrogênio concentraram-se nas frações mais retidas, com quantidades decrescentes nas frações mais próximas da saída.

Esses resultados permitiram que Gilpin e Day confirmassem que o "fracionamento" de diferentes amostras de petróleo pela argila era viável. Entretanto, eles nunca obtiveram a "separação completa" dos componentes de petróleo, prevista por Day em sua palestra em Paris.

Os trabalhos de Engler foram continuados por Leo Obbelohde (1876-1964), outro importante químico de petróleo que lecionava na Universidade Técnica de Karlsruhe. Os seus resultados, combinados com os de Endler e 
Tabela 2 Algumas propriedades físico-químicas de frações de uma amostra de petróleo cru, coletadas utilizando o sistema mostrado na Figura $4^{[5]}$.

\begin{tabular}{cccc}
\hline $\begin{array}{c}\text { Tubo da } \\
\text { amostragem }\end{array}$ & $\begin{array}{c}\text { Volume coletado } \\
(\mathrm{mL})\end{array}$ & $\begin{array}{c}\text { Massa específica } \\
\text { a } 15^{\circ} \mathrm{C}\left(\mathrm{g} \cdot \mathrm{g}^{-1}\right)\end{array}$ & Descrição da fração \\
\hline 1 & $\sim 80$ & 0,7415 & Clara sem cor \\
2 & $\mathrm{n} \cdot \mathrm{m} \cdot \cdot^{\mathrm{b}}$ & $\mathrm{n} \cdot \mathrm{m}$. & Clara sem cor \\
3 & $\sim 40$ & 0,7428 & Clara sem cor \\
4 & 40 & 0,7458 & Clara com leve fluorescência \\
5 & 40 & 0,7500 & Clara com leve fluorescência \\
6 & 60 & 0,7551 & Levamente amarelo com fluorescência \\
\hline
\end{tabular}

${ }^{a} \mathrm{~A}$ amostra foi uma mistura $(1: 1)$ de um óleo de massa específica de $0,8614\left(15^{\circ} \mathrm{C}\right)$ e uma gasolina de massa específica de $0,6467\left(15^{\circ} \mathrm{C}\right)$. ' $\mathrm{Não}$ medido; a fração não tinha volume suficiente.

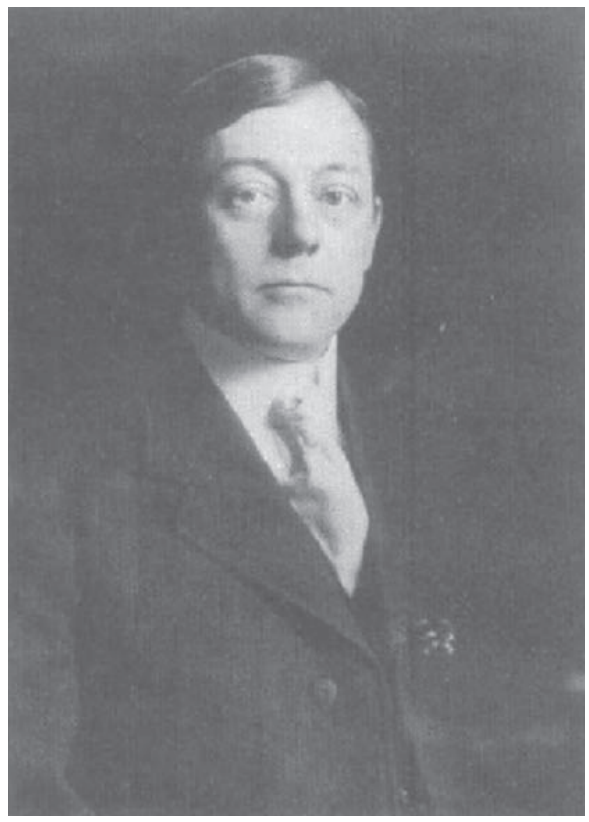

Figura 5 Foto de J.E. Gilpin.

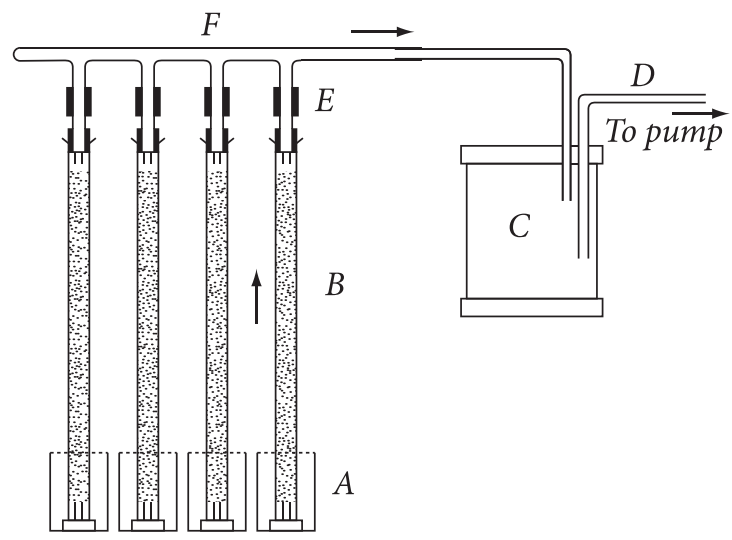

Figura 6 Arranjo experimental de Gilpin e seus alunos ${ }^{10}$.
Albrecht, permitiram que ele propusesse que o mecanismo envolvido no fracionamento de petróleo pelas argilas (além de terra de pisoeiro, várias outras argilas foram avaliadas, com resultados similares) não havia sido devido à "ação capilar" e nem às diferenças em viscosidade, mas, sim, à adsorção diferenciada dos componentes das amostras nos sólidos colocados nos tubos de fracionamento ${ }^{12}$; em outras palavras, o mecanismo envolvido no fracionamento do petróleo pelas argilas foi o de adsorção, proposto por Tswett em $1906^{13}$.

Devido aos campos de exploração de petróleo na Rússia Imperial (mais tarde a União Soviética e hoje Azerbaijan), iniciados nos anos de 1880, alguns pesquisadores russos repetiram os experimentos de Engler e Albrecht na tentativa de aplicar a "filtração" para melhorar as propriedades do petróleo. Um deles, V.E. Herr, descreveu a filtração de um amostra de Baku através de terra de pisoeiro, indicando que esse processo poderia remover a fração pesada de aromáticos policíclicos ${ }^{14}$. É interessante notar que, nesse trabalho, Herr especificamente indicou que o processo não foi de "fracionamento" no sentido de "separação" em componentes individuais, mas simplesmente uma "concentração" de componentes com propriedades similares. 
Tabela 3 Algumas propriedades físico-químicas do líquido extraído de secções da argila após tratamento no sistema mostrado na Figura $5^{[9]}$. Tempo de análise: 23,5 horas.

\begin{tabular}{ccccc}
\hline Fração $^{a}$ & $\begin{array}{c}\text { Distância do topo } \\
\text { do tubo }\end{array}$ & $\begin{array}{c}\text { Volume extraídoc } \\
(\mathrm{mL})\end{array}$ & $\begin{array}{c}\text { Massa específica } \\
\left(\mathrm{g} \cdot \mathrm{g}^{-1}\right)^{\mathrm{d}}\end{array}$ & $\begin{array}{c}\text { Viscosidade } \\
(\text { centipoise })^{\mathrm{e}}\end{array}$ \\
\hline A & $0-31^{f}$ & - & - & - \\
B & $31-39$ & 42 & 0,796 & 0,0376 \\
C1 & $39-47$ & 45 & 0,808 & 0,0529 \\
C2 & $47-65^{9}$ & 75 & 0,8125 & 0,0501 \\
D & & 24 & 0,9137 & 0,0529 \\
E & $65-95$ & 130 & 0,815 & 0,0504 \\
F & $95-130$ & 170 & 0,818 & 0,0521 \\
\hline
\end{tabular}

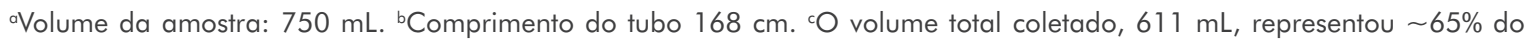
volume aplicado. 'Massa específica da amostra original: 0,810 . ${ }^{e}$ Viscosidade relativa à água. ${ }^{\circledR}$ Nenhum óleo atingiv esta parte da argila. "'Esta fração foi extraída duas vezes.

Um outro russo, M.A. Rakusin, repetiu alguns dos experimentos de Endler e Albrecht com amostras de Baku e, diferentemente do seu compatriota, propôs separação por ação capilar ${ }^{15}$.

Outros pesquisadores na área de petróleo continuaram a aplicação desse processo, hoje identificado como cromatografia líquido-sólido (CLS), na tentativa de obterem frações mais puras e identificarem, com melhor precisão, os seus constituintes. A maioria das aplicações de CLS foram em escala preparativa na tentativa de isolar compostos individuais. Um projeto que merece ser citado foi o Projeto No. 6 do Instituto Americano de Petróleo (API). Iniciado em 1927, esse projeto continuou até 1967, utilizando uma “amostra padrão" de petróleo, obtida de um poço localizado no estado de Oklahoma. Após várias tentativas de aplicar processos de extração e de destilação, os pesquisadores iniciaram separações cromatográficas, com colunas recheadas com sílica gel e eluídas com diversos solventes orgânicos ${ }^{16}$. Na tentativa de se obterem melhores separações aplicando a CLS, um conjunto de colunas de aço inoxidável de $1600 \times 2 \mathrm{~cm}$ foram construídas em um poço de elevador, cada uma recheada com $3,7 \mathrm{~kg}$ de sílica gel (Figura 7) ${ }^{17}$. Tipicamente, uma amostra de 0,5 L foi aplicada no topo da coluna. Quando toda a amostra entrou na coluna, a eluição, usualmente com etanol, foi iniciada. $\mathrm{R}_{1}$ e $\mathrm{R}_{2}$ correspondem aos reservatórios para os eluentes. O sistema pode ser aquecido com um fio aquecedor colocado ao redor da coluna e mantido em atmosfera de nitrogênio (porém, nenhuma menção do uso de pressão do gás para apressar a separação foi indicada). Recipientes colocados no fim da coluna (C) coletaram as frações. Quando se completou cada separação, indicada pela presença de etanol (ou outra fase móvel) puro no frasco de coleção, a sílica foi regenerada passando nitrogênio, de baixo para acima, a uma temperatura de $200{ }^{\circ} \mathrm{C}$, recuperando a fase móvel no frasco $\mathrm{R}_{3}$. Com uma amostra do "petróleo padrão", foram obtidas 175 frações com propriedades diferentes, em uma separação que durou 45 dias. As colunas também foram utilizadas em separações de diversas frações obtidas por destilação. Em uma dessas, os isômeros 1-metilnaftaleno e 2-metilnaftaleno foram separados, ilustrando a alta eficiência (para o ano em consideração) dessas colunas longas ${ }^{17}$. 


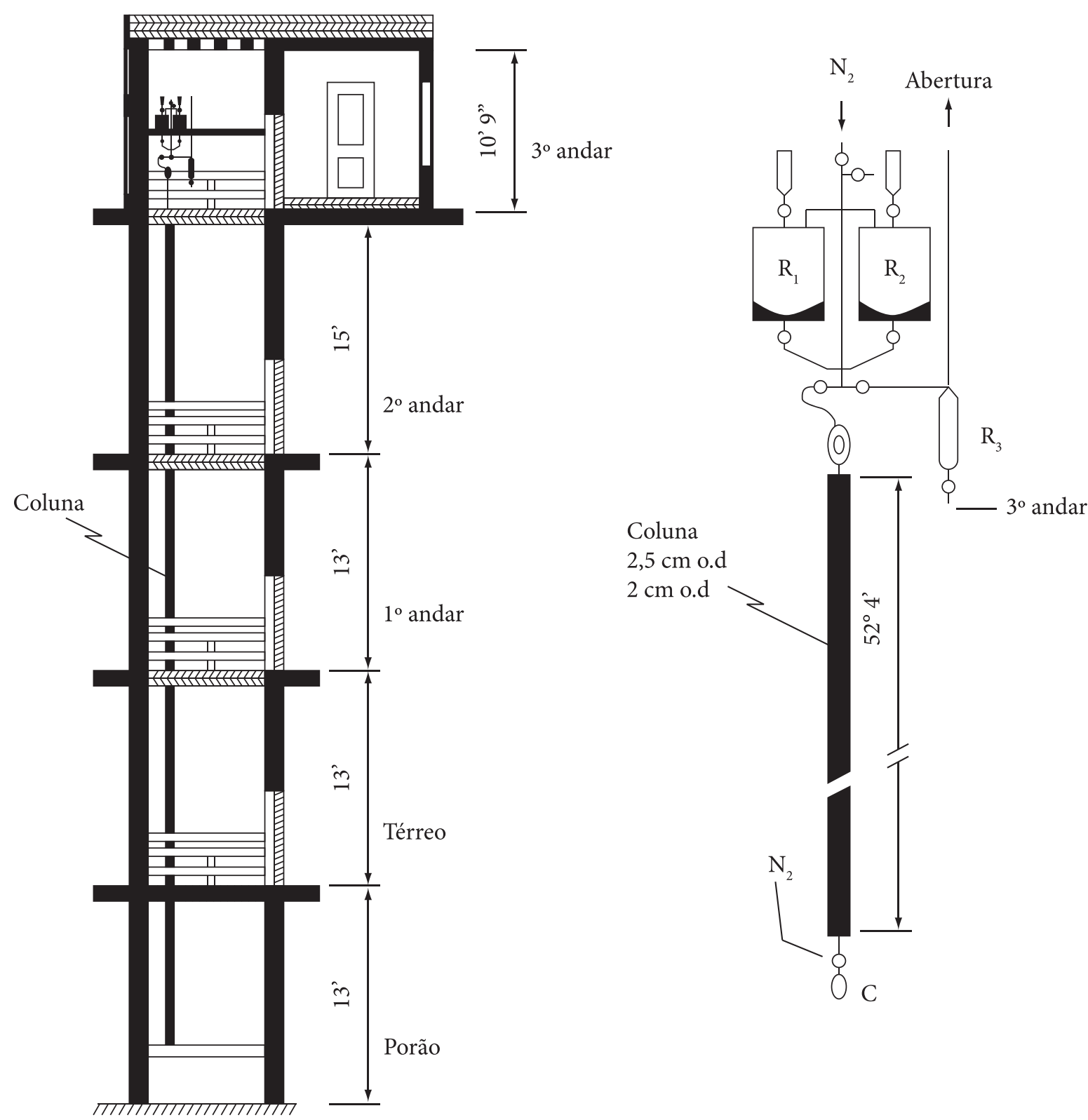

Figura 7 A longa coluna de CLS do Projeto No. 6 do API.

\section{Referências}

1 Engler C, Böhm M. Über den chemische Natur des Vaselins. Dingler's Petrotechnology Journal 1886; 262:468.

2 Engler C, Böhm M. Über den chemische Natur des Vaselins. Dingler's Petrotechnology Journal 1886; 262:524.

3 Day DT. A suggestion as to the origin of Pennsylvania petroleum. Proceddings of the American Philosophical Society $1897 ; 36: 112$.
4 Day DT. La variation des caractères des huiles brutes de Pensylvania et de l'Ohio Resumos do Congrès International du Pétrole; 1900; Paris. Jornal du Pétrole 1902; 1:53.

5 Engler C, Albrecht E. Über den Vorgang bei der Filtration von Petroleum durch Floridaerde. Zeitschrift für Angewandte Chemie 1901; 14:889.

6 Goppelsroeder F. Capillaranalyse beruhendvauf Capillaritäts- und Adsorptionserscheinungen. Basel: Emil Birkhäuser Verlag, Basel; 1901. 
7 Collins CH. O desenvolvimento da cromatografia em papel. Scientia Chromatographica 2009; 1(4):7.

8 Day DT, Gilpin JE. The changes in crude petroleum effected by diffusion through clay. Industrial \& Engineering Chemistry 1909; 1(7):449-455. doi: 10.1021/ie50007a008.

9 Gilpin JE, Cram MP. The fractionation of crude petroleum by diffusion through fuller's earth. American Chemistry Journal 1908; 40:537.

10 Gilpin JE, Bransky OE. The diffusdion of crude petroleum through fuller's earth. American Chemistry Journal 1910; 44:251.

11 Gilpin JE, Schneeberger P. Fractionation of California petroleum by diffusion through fuller's earth. American Chemistry Journal 1913; 50:59.
12 Ubbelohde L. Bemerkungen $\mathrm{zu}$ einigen Arbeiten Rakusins über das geologische Alter ynd über die optische Aktivität des Erdöis. Petroleum (Berlin) 1909; 4:1394.

13 Collins CH. Michael Tswett e o "nascimento" da cromatografia. Scientia Chromatographica 2009; 1(1):1.

14 Herr VF. Zur Kenntnis der Filtration von Baku-Erdölen durch Fullererde. Petroleum (Berlin)1909; 4:1284.

15 Rakusin MA. Petroleum (Berlin)1910; 5:760.

16 Mair BJ, White JD. Journal of Research of the National Bureau of Standards 1935; 15:51.

17 Mair BJ, Gaboriault AL, Rossini FD. Assembly and testing of 52-foot laboratory adsorption column. Industrial \& Engineering Chemistry 1947; 39(9):1072-1081. doi: 10.1021/ie50453a003. 